\title{
Prevention and Management of Complications Resulting from COMMON SPINAL InJECTIONS
}

\author{
Robert E. Windsor, MD, Seneca Storm, MD, and Ross Sugar, MD
}

When spinal injections are performed, a needle is placed in or around the spine making the risk of complications unavoidable. Spinal structures or adjacent organs are at risk for direct needle trauma, infec tion, hematoma, hemorrhage, nerve dam-

Spinal injections are being performed with increasing frequency in the management of acute and chronic pain syndromes (1-4). Since these procedures require a needle to be placed in or around the spine, there is always a risk of complications. For this reason, knowledge of prevention and the early recognition and management of complications is critical in order to deliver appropriate patient care. This review will discuss patient preparation, patient monitoring, and specific complications and their treatment.

\section{Patient Preparation}

Patient preparation includes patient education, informed consent, NPO status, intravenous access, ascertaining that no procedural contraindications exist, patient positioning, sterile preparation and draping, supplemental IV fluids and oxygen, and appropriate recovery following the procedure. Depending on the procedure and the patient status, prophylactic antibiotics may also be indicated.

Patient education should include a thorough description of the procedure including potential risks, benefits, alternatives, and likely outcome. An informed consent should be executed that reflects the conversation. Execution of the informed consent should include signature by the patient, the doctor, and a wit-

From Georgia Pain Physicians and Emory University Atlanta, Georgia. Address Correspondence: Robert E. Windsor, MD, Georgia Pain Physicians, 2550 Windy Hill Rd., Suite 215, Marietta, Georgia- 30067. E-mail: rwindsor@aol.com

Funding: There was no external funding in preparation of this manuscript. age, stroke, allergic reaction, or spinal anesthesia with cardiorespiratory arrest. As a result, physician knowledge and patient preparation and monitoring are critical in maximizing patient safety during the procedure being performed. This review de-

ness. A large bore IV (ideally 20G or larger) should be started in a large proximal upper extremity or neck vein. This is to allow immediate IV access in an emergent setting. Small gauge or peripherally placed IV catheters do not allow adequate access to the central venous supply for resuscitative purposes once peripheral vasoconstriction occurs. Procedural contraindications or relative contraindications that may not have been present or recognized during the last physician office visit should be reviewed such as chest pain, shortness of breath, fever, systemic infection, uncontrolled hypertension or other medical problems. If the procedure involves placement of a needle or other instrument through the skin and into a disk or implantation of a device, then pre-procedure laboratory work should be performed. In addition, if the patient is recovering from a known systemic infection (eg, pneumonia or urinary tract infection) then pre-procedure laboratory work should also be performed. If the patient is infirm (eg, COPD, heart disease) then clearance from the patient's primary care or specialty physician should be obtained. Depending on the patient's problem, pre-procedure laboratory work may include a complete blood count with differential, liver function tests, urinalysis, chest X-ray, EKG, blood culture and sensitivity, urine culture and sensitivity, and sedimentation rate.

The patient should be positioned on the procedure table in a comfortable manner that will allow the treating physician unencumbered access to the region of the patient's body under treatment. The pa- scribes complications that may result from commonly performed spinal injections and their treatment.

Keywords: Epidural steroid injection, complications, spinal injection, safety, risk management

tient's position should be comfortable enough for them to lie there for the duration of the procedure. Care must be taken to make certain that there is no region of neural compression or stretch, particularly if sedating medication will be used. Areas that are particularly vulnerable to neural compression or stretch include the ulnar nerve at the elbow and the brachial plexus (5). If necessary, an arm board, tape, strapping, or padding may be used to make them more comfortable, hold the appropriate position, and keep their hands from inadvertently embarrassing the sterile field.

Sterile preparation should minimally include scrubbing the region of the body to be treated and the surrounding areas with a povidone iodine preparation and allowing it to dry. If the patient has an iodine allergy then chlorhexidine gluconate and/or isopropyl alcohol should be used. For discography or any type of implant, a triple scrub which includes isopropyl alcohol, chlorhexidine gluconate, and povidone iodine must be used, with the scrub lasting at least 5 minutes. The povidone iodine is allowed to dry. Although controversial, for these procedures, pre and postprocedure antibiotics may be used.

The degree of sterile draping required depends on the procedure. If a lumbar epidural is being performed then draping the immediate area to be penetrated with sterile towels is adequate. If a spinal implant, percutaneous discectomy, or other more invasive spinal procedure is being performed then full body draping with a fenestrated drape, iodine impregnated adhesive biodrapes, sterile towels, 
and half sheets should be used as needed in order to ensure a sterile field.

Supplemental fluids are important during most procedures whether it is a high risk procedure or not. When the patient has been NPO for 3 hours or particularly during morning procedures when they have been NPO since the night before, they are somewhat volume depleted and are more prone to vasovagal reactions. Supplemental fluids before, during, and after the procedure help prevent such reactions. In addition, it is helpful to have fluids already flowing in the event that the patient becomes hypotensive or to help flush medications through the line. Supplemental fluids should be used cautiously if the patient is volume-sensitive such as in congestive heart failure or renal pathology.

Supplemental oxygen should be directed by the situation. If the patient desaturates for any reason, supplemental oxygen should be used as needed to help maintain the patient's oxygen saturation above $90 \%$. If the patient has chronic obstructive pulmonary disease (COPD) or other pulmonary pathology then supplemental oxygen should be used sparingly as too much oxygen may further suppress their respiratory drive. In addition, if the patient has chronic pulmonary disease the treating physician must confirm that they can tolerate the position required by the procedure.

\section{Patient Monitoring}

Patient monitoring should minimally include blood pressure and heart rate monitoring. If the patient is infirm, a high-risk procedure is being performed, or IV sedation is being used, then cardiac monitoring and pulse oximetry should also be employed. Baseline vital signs should be obtained before the procedure for purposes of comparison during and after the procedure. Pre-procedure hypertension should be approached with caution. A patient with cerebrovascular disease may require a higher than normal blood pressure to maintain cerebral perfusion, thus adjusting their blood pressure may incite a stroke. If it is deemed that lowering their blood pressure is medically safe and appropriate then gentle IV sedation is generally all that is required. Sublingual calcium channel blockers should be avoided (6). In addition, if IV sedation for the procedure is planned then blood pressure reduction with other med- ications should not be carried out prior to sedation as this combination of drugs may lower the blood pressure to dangerous levels.

Cardiac monitoring should be employed with any patient with a significant cardiovascular history or when known risks of the procedure planned may place the patient at risk for cardiovascular complication. In general, cardiac monitoring should be performed in any patient with a known history of myocardial infarction or angina; a significantly invasive procedure (eg, spinal implant); an intraspinal cervical or thoracic procedure; a procedure that may place a significant volume of local anesthetic or narcotic in the spinal canal or systemic circulation; or a procedure that will require a significant amount of IV sedation.

\section{Patient Recovery}

The recovery of the patient following the procedure is critically important and is often the part of the procedure that is ignored. The post-procedural period is the time in which most procedure-related complications occur. Complications that occur during the immediate post-procedure period include hypotension, vasovagal reactions, sensorimotor blockade, excessive somnolence, respiratory suppression, seizures, and cardiovascular events.

For these reasons it is important to have a medically reasonable recovery protocol that ultimately allows for the patient to be recovered in a monitored situation until they are alert; oriented; tolerating fluids, and ambulating safely. Ideally, their sensorimotor status should return to their pre-morbid condition. This may be unreasonable if a long acting local anesthetic has been injected into the spinal canal.

The patient is transferred from the operating room or procedure room in the recumbent position or in a wheelchair to recovery area. They are observed with intermittent vital sign and continuous pulse oximetry monitoring. If the patient received no conscious sedation during the procedure then they are required to stay in the recovery area for at least an additional 15 minutes or until they have met the above outlined criteria. If the patient received IV sedation during the procedure, then they must remain in the recovery area for 20-60 minutes following the last dose of sedating medication or outlined criteria; and they must have some- one else drive them home.

If the specific intervention was more significant than a simple spinal injection (eg, spinal implant, percutaneous discectomy) then the recovery period may last for up to 8 hours. Once they have met discharge criteria, they are discharged with appropriate safety and follow-up instructions.

\section{General Complications of Spinal INJECTIONS}

\section{Infectious Complications}

Infections occur in $1-2 \%$ of spinal injections ranging from minor to severe conditions such as meningitis $(7,8)$, epidural abscess (9-11), and osteomyelitis $(12,13)$ (Figs 1 and 2). Severe infections are rare and occur between 1 in 1,000 and 1 in 10,000 spinal injections. Severe infections may have far reaching sequelae such as sepsis, spinal cord injury, or spread to other sites of the body via Batson's plexus or direct contiguous spread. Poor sterile technique is the most common cause of infection. Staphylococcus aureus is the most common offending organism causing infection that is picked up from skin structures. Infection from gram-negative aerobes and anaerobes may occur from inadvertent intestinal penetration. Usually, discitis from lumbar discography involves a gram-negative aerobe, is self-limited and resolves with early recognition and administration of appropriate anti-

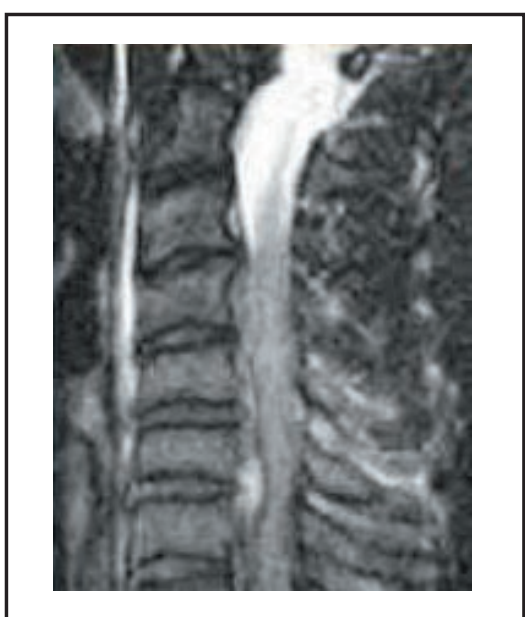

Fig. 1. Cervical Epidural Abscess (MRI view). T2-weighted image demonstrating an epidural abscess compressing the thecal sac and spinal cord at C5, C-6 and C-7 levels. The actual abscess extends from C5 to T1. 


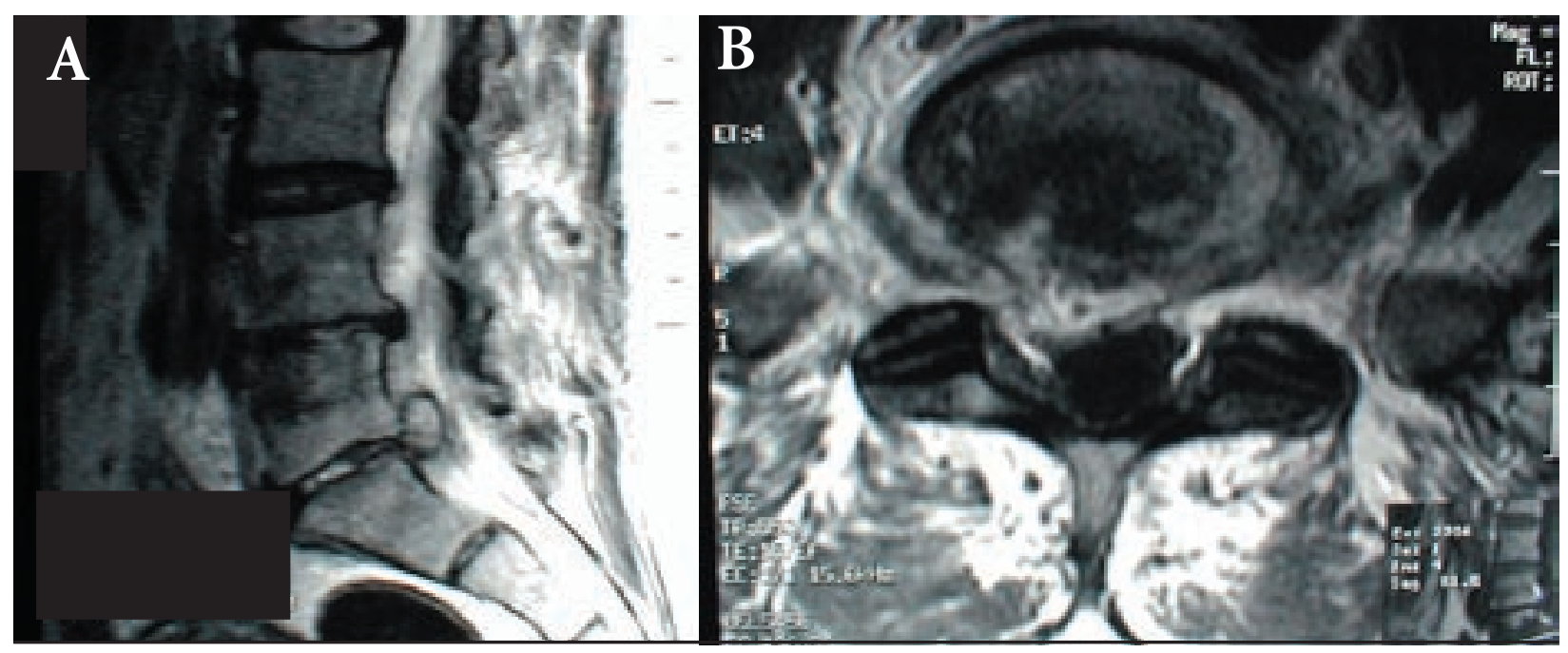

Fig. 2. L5-S1 Discitis with Epidural Abscess (MRI view).

A. T2-weighted sagittal MRI image demonstrating increased signal intensity within the L5-S1 disc, an adjacent high intensity oval lesion within the spinal canal, and high intensity changes in the adjacent L5 and S1 vertebral bodies. These changes represent an L5-SI discitis with an adjacent epidural abscess compressing the right anterolateral thecal sac, L5 and S1 nerve roots with vertebral body marrow edema.

B. This image is an axial image of the L5-S1 disc shown in sagittal section demonstrating compression of the right anterolateral thecal sac and L5 and S1 nerve roots.

biotics. Cervical discitis, however, is often life- threatening due to the aggressive gram-negative anaerobes that colonize the esophagus. If the infection is a mild cutaneous infection and the patient is immunocompetent then it will usually resolve with local cleaning with antiseptic agents. The physician should make specific hygiene recommendations and follow this infection expectantly. If it appears to be pursuing a more aggressive course but does not appear to involve spinal structures then appropriate oral antibiotics on an outpatient basis and frequent follow up may be all that is required. If the infection appears to be progressing to spinal structures or spaces, or if the patient is infirm or otherwise predisposed to infection, then in-patient evaluation and care with appropriate IV antibiotics is usually required. If epidural abscess occurs, emergent surgical drainage must be considered to avoid neural damage or other complications (14).

Early detection and treatment of epidural or intrathecal infection is necessary in order to avoid morbidity and mortality. It usually presents with severe back or neck pain, fever, and chills with a leukocytosis developing on the third day following the injection (11). Patients with diabetes or other immunocompromising conditions are more susceptible to infec- tion and should be followed very closely following spinal injections. In these patients, once infection is suspected or confirmed then they must be evaluated and treated aggressively. Pre-existing systemic infection is a relative contraindication to spinal injection. If the spinal injection is critical to the overall care of the patient with pre-existing systemic infection then the risks and benefits must be carefully weighed prior to performing the injection. In this clinical setting, prophylactic antibiotics should given to the patient for 72 hours or until the patient has been afebrile for at least 24 hours prior to performing the injection. It is important to know what the standard of care for preventing or treating spinal injection-related infections is in your area are and to routinely review current micro-organism susceptibilities.

\section{Cardiovascular Complications}

Bleeding is a risk inherent to all injection and surgical procedures. The potential for bleeding during spinal injection is increased by liver disease, the consumption of Coumadin or other anti-coagulants, certain inherited anemias such as G6PD deficiency or sickle cell anemia, coagulopathy from whatever cause and venous or arterial puncture. The epidural vasculature is injured in $0.5-1 \%$ of spinal injections on average and is more common with placement of the needle in the lateral portion of the spinal canal than the midline (15-17). It has been our experience that placing bolsters under the patient allowing the belly to hang in a pendulous manner substantially evacuates Batson's plexus. Significant epidural bleeding may cause the development of an epidural hematoma (Fig. 3). Clinically significant epidural hematomas are rare with a reported incidence of between 1 in $4,000(18,19)$ and 1 in 10,000 lumbar epidural cortisone injections and may lead to irreversible neurologic compromise if not surgically decompressed within 24 hours of symptom onset $(15,18-24)$. Retroperitoneal hematomas may occur following spinal injection if the large vessels are inadvertently penetrated. These hematomas are usually self-limited but may be a cause of acute hypovolemia or anemia.

In addition to bleeding, a variety of dysrhythmias may occur. When a dysrhythmia occurs, treatment should be initiated immediately. It is important that the entire medical care team (MCT) is able to function synergistically when treating a dysrhythmia. Advanced cardiac life support (ACLS) code scenarios should be implemented. Treatment of individual dysrhythmias are beyond the scope of this article; however, the reader is directed to 


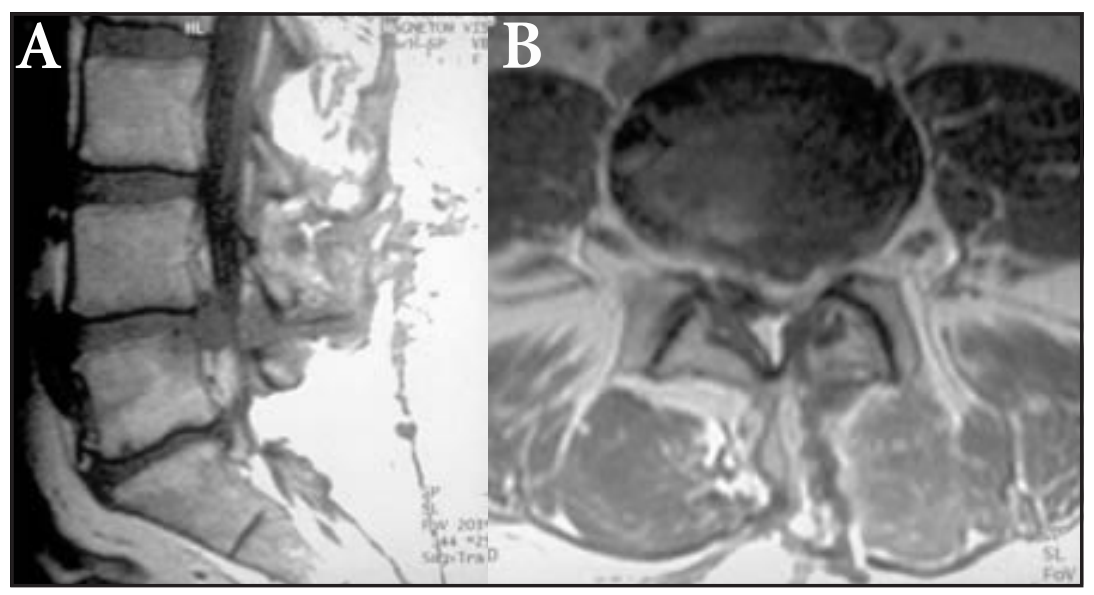

Fig. 3. Acute Epidural Hematoma (MRI view).

A. T2-weighted sagittal MRI image demonstrating an acute epidural hematoma at the L5-S1 level compressing the left anterolateral thecal sac and $L 5$ and $S 1$ nerve roots.

B. T2-weighted axial image demonstrating a small low intensity mass within an encompassing high intensity mass compressing the left anterolateral thecal sac and left L5 and SI nerve roots.

the Emergency Cardiac Care Algorithms (25) and other sources for more detailed information $(26,27)$.

\section{Neurological Complications}

Neurological complications are rare. The most common causes of neural injury during spinal injection is direct trauma to the spinal cord or nerve roots from a needle, compression from an epidural hematoma, or from involvement by infectious exudate. Brain injury may occur from protracted seizure activity lasting longer than 30 minutes (28-30), ether procedure induced, sedation-induced or cardiac-induced hypotension; the dislodgement of plaque from intra-arterial injection with embolism, or anoxia from respiratory arrest or laryngeal obstruction. The proximity of the vertebral artery during cervical trans-foraminal or facet joint injections requires particular knowledge of the three-dimensional anatomy and radiographic anatomy of the cervical spine as well as specific training and expertise in cervical spinal injection procedures in order to consistently protect these structures. Injection into this vessel may cause a posterior circulation stroke, hematoma formation and occlusion of the vessel. Studies demonstrate that fluoroscopically guided spinal injections are less apt to cause inadvertent neural injury, injection into a vascular structure, and provide improved precision of needle placement (16,
17, 31-35). A pertinent neurologic review of symptoms and physical exam should be performed immediately if a neurologic complication is suspected.

\section{Respiratory Complications}

Respiratory arrest is defined as apnea for greater than one minute due to lack of central respiratory drive or paralysis of the muscles of respiration (36). Respiratory arrest may occur from a variety of causes including over sedation, central nervous system trauma, and the intrathecal or epidural injection of a sufficient amount of local anesthetic to cause spinal anesthesia. Treatment includes the immediate recognition of the condition and emergent support of vital signs. If the cause is self-limited the treatment may require the support of respiration and other vital signs as needed until spontaneous and adequate respiration resumes. If the cause may last for a prolonged period of time and can be easily reversed then it should be such as when too much narcotic or sedative has been given. In this clinical setting it is important to keep in mind the half-life of the reversing agent as compared to the half-life of the narcotic or sedative being reversed. If the narcotic or sedative's half-life is longer than that of the reversing agent then respiratory compromise may resume once the reversing agent has been metabolized.

The true incidence of respiratory de- pression due to spinal opioid administration is unknown. Factors that may cause respiratory depression are the use of sedative, parenteral or spinal opioids, and parenteral or spinal local anesthetics. One of the main advantages of spinal versus parenteral opioid administration is the lack of respiratory depression with the former (36-39).

Other respiratory complications due to spinal injections include pneumothorax and injury to the recurrent laryngeal nerve. A pneumothorax may occur during a lower cervical procedure such as a discogram or selective nerve root block or a thoracic procedure such as an intercostal nerve block. As a general rule, a pneumothorax may not occur if a needle penetrates the pleural cavity or lung parenchyma unless it is placed through a bleb, the needle is an 18 gauge or larger, or a solution has been injected. When a pneumothorax does occur it is usually self-limited and cause only minor (10\%) collapse of the lung $(40,41)$. Treatment includes close observation with supportive care, usually in a hospital, and serial chest Xrays. A chest tube should be placed if the pneumothorax advances significantly over $25 \%$ or the patient develops shortness of breath or other signs of respiratory distress (41).

Injury to the recurrent laryngeal nerve may cause unilateral vocal cord paralysis, reduction in the ability to protect the airway, and hoarseness. This injury is usually self-limited and resolves on its own but may be clinically significant while the patient is recovering from sedation or if there is pre-existing underlying pathology that causes marginal airway protection (eg, stroke, laryngeal cancer).

\section{Urological Complications}

The application of local anesthetics and/or opioids to the lumbar and sacral nerve roots results in higher incidence of urinary retention (42). This side effect of lumbar epidural nerve block is seen more commonly in elderly males, multiparous females, and patients who have undergone inguinal and perineal surgery. Overflow incontinence may occur if such a patient is unable to urinate or bladder catheterization is not utilized. All patients should urinate prior to undergoing lumbar epidural nerve block and should demonstrate the ability to urinate prior to discharge from the pain center. 


\section{Dural Puncture}

In the hands of the experienced interventional spine specialist, inadvertent dural puncture during lumbar epidural injections should occur in $<0.5 \%$ of cases (or 1 in 200 epidural injections) (43). Positional headache may result when the thecal sac is violated by the epidural needle and a sufficient amount of cerebrospinal fluid (CSF) has escaped allowing the CSF pressure to drop enough to permit the brain to decrease in volume. This reduction in volume causes the length sensitive nociceptors to become electrically excited. The CSF pressure drops most when the patient raises their head above heart level such as in sitting or standing, which may cause a severe throbbing headache when in these positions (44-48). The occurrence of postdural puncture headache is an annoying side effect, but is generally relatively benign and will pass without permanent harm or morbidity to the patient. Rarely, with dehydration and severe nausea and vomiting, uncal herniation may occur with associated brainstem involvement and potentially death (48). If a needle is placed subdurally and epidural doses of local anesthetics are administered the signs and symptoms are similar to subarachnoid injection (49). The subdural or subarachnoid injection of large doses of local anesthetics may cause total spinal anesthesia, loss of consciousness, hypotension, cardiovascular arrest, apnea, and death. This condition requires immediate resuscitative measures and support of all vital signs until the condition resolves. Intubation is usually required to adequately control the airway and ventilate the patient.

\section{Fluoroscopic Exposure}

Intended epidural injections performed without fluoroscopy are not always optimally placed and/or the medication does not get to the desired target organ due to anatomic abnormality as noted in various sources $(31,32,34,50-54)$. For this reason, most spine management specialists recommend fluoroscopic direction and the use of non-ionic or low ionic contrast agents for epidural injections. This helps confirm accurate needle placement and the delivery of the injected solution to the appropriate target organ. The risk of fluoroscopic exposure to the patient is minimal for one or several isolated procedures due to the low dose of ionizing radiation delivered to patients from prop- erly calibrated digital fluoroscopy equipment (55-60).

Exposure to the physician, attending nurse, radiological technologist and anyone else consistently in the procedure room should be viewed as being cumulative. To limit exposure to the physician, patient, and other personnel, it is important to note that radiation dissipates at the inverse of the square of the distance from the tube. As a result, standing six feet or more from the fluoroscopic tube during active fluoroscopy reduces the risk of excessive exposure. The fluoroscopy anode should also be kept under the procedure table as this is the source of the radiation. In this way, the patient absorbs the bulk of the directed radiation and the majority of the small amount of radiation spilled into the room, known as "scatter radiation", has significantly less energy than directed radiation and has a substantially reduced ability to penetrate tissues. The physician should wear a lead apron, thyroid shield, radiation attenuating gloves, and perhaps lead-lined glasses. The nurse and radiological technologist should wear wrap around lead aprons as their back is frequently turned to the radiation source and a thyroid shield. All personnel should wear radiation badges under their thyroid shield and apron, on top of their thyroid shield to measure the radiation exposure to their face, and the physician should consider wearing a ring badge if their hand is routinely in the field during active fluoroscopy. The use of radiation-attenuating gloves is somewhat controversial since the placement of the lead-lined gloves in the fluoroscopic field will cause the fluoroscopy unit to automatically increase its radiation exposure subjecting the patient and the personnel to increased radiation exposure. Additional protective measures with lead drapes from table to floor may also be considered (60). If the physician decides to use radiation attenuating gloves then the gloved hands should be in the radiation field for very brief periods of time (1-2 seconds) if at all. This minimizes the fluoroscopy unit's ability to adjust and increase its energy output. Finally, the fluoroscopy unit must be routinely maintained and inspected to confirm its proper function and safety. With the proper use of fluoroscopy and radiation safety, the use of fluoroscopy to direct and confirm proper needle placement should maximize the benefit while limiting potential risks to the patient, physi- cian, or personnel.

\section{Medication Reactions}

Adverse drug reactions are rarely seen with medications used during spinal injections. The treating physician should be aware of drug toxicity, side effects, allergic reactions, concentration, and dosing of all medicines used. Lidocaine and bupivacaine are the most common local anesthetics used during spinal injections. It is important to be aware of their CNS and cardiovascular toxicity and side effects. Strict cardiovascular and neurological monitoring is required, before, during, and after the procedure. Although most anaphylactic reactions occur most often within 2 hours after the epidural injection, they have been known to occur up to 6 hours later (61).

Local anesthetics primarily function by reversibly blocking the sodium channels in nerve and muscle membranes having a direct effect on sympathetic nerves when injected into the subarachnoid space and the cardiac tissue when injected intravascularly. If the sympathetic system is sufficiently blocked then hypotension may result and if cardiac muscle is sufficiently blocked then decreased contractility may result. When injected intravenously, lidocaine is fast-in and fast-out reaching steady-state in one to two beats. Bupivacaine is fast-in and slow-out and its blocking action increases as the heart works harder. These are the main direct effects that may cause cardiac arrest. Cervical and thoracic level blocks have an increased risk for complications due to the regional cardiac and respiratory control centers.

Central nervous system toxicity by $1 \%$ lidocaine has an onset at plasma concentrations of $5-10 \mathrm{ug} / \mathrm{ml}$ which is slightly more than $400 \mathrm{mg}$ (or $40 \mathrm{cc}$ ) of total intravenous bolus and bupivacaine is about four times more toxic than lidocaine with a toxic bolus of $100 \mathrm{mg}$ (or $10 \mathrm{cc}$ ) (62). A person with central nervous system (CNS) toxicity usually presents with complaints of circumoral numbness, disorientation, light-headedness, nystagmus, tinnitus, and muscle twitching in the face or distal extremities. Peak-plasma concentrations occur 10-20 minutes after injection. Thus, patient monitoring for at least 30 minutes following an epidural injection with a significant bolus of lidocaine or bupivacaine is mandatory.

Methylprednisolone, triamcinolone, 
and betamethasone are the most commonly used corticosteroid preparations (63). Side effects are uncommon but include headaches, dizziness, insomnia, facial erythema, rash and pruritus, lowgrade "fever" (< I00F), hyperglycemia, transient hypotension and hypertension, increased back or limb pain, fluid retention, mood swings, euphoria, menstrual irregularities, headaches, and gastritis. Other rare side effects include elevation of cerebrospinal fluid protein levels, septic or aseptic meningitis, worsening of symptoms of multiple sclerosis, sclerosing spinal pachymeningitis, exacerbation of latent infection, near-fatal septic meningitis (intrathecal injection), hypercorticism, and congestive heart failure.

\section{Anaphylactic and Allergic Reactions \\ Anaphylactoid (without histologic immune response) or anaphylactic (with a histologic immune response) reactions occur most often within 2 hours after the epidural injection and have been known to develop up to 6 hours later (61). This usually causes fatalities by respiratory-re- lated complications involving mechanical airway obstruction. Close patient moni- toring after the procedure is recommend- ed for approximately 30 minutes. Inform- ing the patient of possible risks would ex- pedite early identification of complica- tions.}

\section{Specific Complications of Spinal INJECTIONS}

\section{Lumbar Epidural Injections}

The lumbar epidural space is highly vascular. Inadvertent intravenous placement of the epidural needle occurs in approximately $0.5 \%$ to $1 \%$ of patients undergoing lumbar epidural anesthesia (43). This rare complication is mostly seen with distended epidural veins such as occurs during pregnancy and in patients with a large abdominal tumor mass. If the misplacement is unrecognized, injection of a large volume of local anesthetic directly into an epidural vein may result in significant local anesthetic toxicity (64). Careful four-quadrant aspiration (aspiration in all four quadrants by rotating the needle) prior to injection of drugs into the epidural space is mandatory in identifying the vascular placement of the needle when performing a "blind" (non-fluoroscopically guided) epidural injection. If fluoroscopy is used, the injection of contrast will usually identify any significant intravascular injection.

Neurologic complications of lumbar nerve block are uncommon if proper technique is used. Usually, these complications are associated with a preexisting neurologic lesion or with surgical or obstetric trauma rather than with the lumbar block itself (42). Direct trauma to the spinal cord or nerve roots is usually accompanied by pain. Any significant pain that occurs during placement of the epidural needle or catheter or during injection should warn the injectionist to pause and confirm needle placement prior to proceeding (43). The use of deep intravenous sedation or general anesthesia prior to initiation of epidural nerve block may reduce the patient's ability to provide accurate verbal feedback if needle misplacement occurs. As a result, conscious sedation or general anesthesia prior to epidural nerve block should be employed with caution $(64,65)$.

When the patient's lower extremity neurologic status deteriorates rapidly or when a cauda equina syndrome is suspected within 24 to 48 hours following an epidural procedure, an expanding epidural hematoma should be considered (66). If the injectionist considers the diagnosis then an immediate and complete clinical evaluation is mandatory. If the diagnosis is still entertained following the clinical evaluation then a lumbar CT scan or MRI should be obtained (Fig. 4). If the diagnosis is confirmed then an emergent surgical consult to consider decompression should be arranged.

\section{Caudal Epidural Injections}

Incorrect needle placement during caudal epidural injection occurs 25 $40 \%$ of the time (51-54). The needle may be placed outside the sacral canal resulting in the injection of air or fluid into the subcutaneous tissues, periosteum, sacrococcygeal ligament, sacral marrow cavity, and pelvic cavity possibly entering both the rectum or vaginal vault. The application of local anesthetic and opioids to the sacral nerve roots results in an increased incidence of urinary retention, especially noted in elderly males and multiparous females and after inguinal and perineal surgery. The use of smaller doses of local anesthetic will help avoid these burdensome complications without adversely affecting the efficacy of caudal epidural steroid injections when treating painful

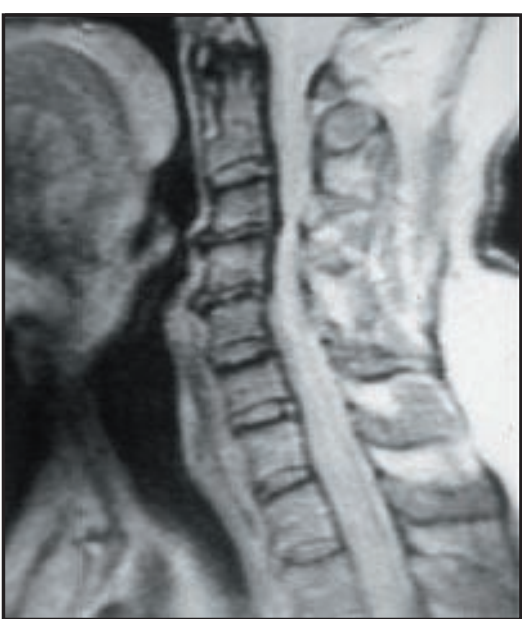

Fig. 4. Cervical Stenosis (MRI view).T2-weighted sagittal MRI image demonstrating congenital stenosis at C3 and C5.

conditions. Because of the proximity of the sacral hiatus to the perineum, there is an increased incidence of epidural abscess and meningitis compared to the interlaminar or transforaminal injection route. When placing the epidural needle, it is important to remember that the thecal sac usually ends at the S2 bony level but it may end as low as S4 thus, the needle should be placed no higher than absolutely necessary to assure epidural injection. If the needle penetrates the thecal sac, this may cause a positional headache and will lower the body's protection against meningitis. In addition, if this needle malposition is not detected prior to injection then an intrathecal injection may occur potentially causing a spinal block and its associated sequelae.

\section{Cervical Interlaminar Epidural Injections}

Because of the potential for hematogenous spread via Batson's plexus, local infection and sepsis represent absolute contraindications to the cervical approach to the epidural space. Anticoagulation and coagulopathy represent absolute contraindications to cervical epidural nerve block because of the risk of epidural hematoma.

The fact that the spinal cord is present within the spinal canal means that there is an increased risk for spinal cord injury with the interlaminar injection technique as compared to lower or mid lumbar injections. Central canal stenosis from bony eburnation, central disc 
Table 1. Treatment of Acute reactions:

- Urticaria

- Discontinue injection

- Benadryl or Vistaril, PO/IM/IV, 25-50 mg

- Cimetidine PO/IV, 30omg or Ranitidine PO/IV, $50 \mathrm{mg}$

- If severely disseminated, then give Epinephrine SC (1:1000), 0.1-0.3 ml

- Facial and Laryngeal Edema

- Epinephrine SC (1:1000), 0.1-0.2 ml, or if hypotensive give 1:10,000, slowly IV, $0.1 \mathrm{ml}$

- *0xygen via mask/endotracheal tube, 6-10 L/min

- If resuscitation needed, initiate ACLS protocol and call EMS (ambulatory setting) or call a code (hospital setting)

- Bronchospasm

- *0xygen via mask, 6-10 L/min

- Monitor vital signs (BP, pulse ox, and EKG)

- Beta agonist inhalers (eg. Albuterol)

- Epinephrine SC (1:1000), 0.1-0.2 ml, or if hypotensive give 1:10,000, slowly IV, $0.1 \mathrm{ml}$

- If oxygen saturations persist $<\mathbf{8 8} \%$, initiate ACLS protocol and call EMS (ambulatory setting) or call a code (hospital setting)

- Hypotension with Tachycardia

- Reverse Trendelenburg position

- Monitor vital signs (BP, pulse ox, and EKG)

- *Oxygen via mask, 6-10 L/min

- Rapid administration of large volumes, IV of isotonic Ringer's lactate or normal saline solution

- If poorly responsive; Epinephrine SC (1:10,000), $1.0 \mathrm{ml}$, slowly IV

- Hypotension with Bradycardia - Vagal Reaction

- Reverse Trendelenburg position

- Monitor vital signs (BP, pulse ox, and EKG)

- *Oxygen via mask, 6-10 L/min

- Secure IV access and initiate rapid administration of large volumes, IV of isotonic Ringer's lactate or normal saline solution

- If poorly responsive; Atropine 0.6-1.0 mg, slowly IV

- Repeat Atropine to a total dose of $0.04 \mathrm{mg} / \mathrm{kg}(2-3 \mathrm{mg})$ in an adult

- Hypertension, Severe

- Monitor vital signs (BP, pulse ox, and EKG)

- Nitroglycerin, 0.4mg, SL or Nitropaste topical ointment, 1-2 inch

- If persistent, transfer for further evaluation to an ER or ICU setting

- For pheochromocytoma; give phentolamine $5 \mathrm{mg}$ (adults), 1mg (children)

- Seizures-Convulsions

- Monitor vital signs (BP, pulse ox, and EKG)

- *Oxygen via mask, 6-10 L/min

- Maintain IV access

- Protect patient from physical injury during seizure

- Insert bite block

- If the seizure is greater than 2 minutes, secure airway and oxygenate

- Obtain neurological consult

- Give Diazepam (Valium) 5 mg, IV or Midazolam (Versed) $2.5 \mathrm{mg}$, IV

- If longer effect needed, consider Phenytoin (Dilantin) infusion 15-18 mg/kg at rate of $50 \mathrm{mg} / \mathrm{min}$

- Consider ACLS protocol, if intubation is needed

- Pulmonary Edema

- Elevate the torso; rotating tourniquets (venous compression)

- *Oxygen via mask, 6-10 L/min

- Diuretics - furosemide (Lasix), 40mg IV, slow push

- Consider morphine

- Transfer to a ICU or ER setting, for further management

Prophylaxis for Adverse Intravascular lodinated Contrast Media Reactions

- Avoid unnecessary exposure to contrast medium

- Substitute non-ionic for ionic contrast medium

- In adults, give prednisone $50 \mathrm{mg}, \mathrm{PO}, 12 \mathrm{hrs}$ then 2 hrs prior to procedure

- Give diphenhydramine (Benadryl), $50 \mathrm{mg}, \mathrm{PO}, 1$ hour prior to procedure

- For pheochromocytoma; give phenoxybenzamine 10- $20 \mathrm{mg}, 3-4$ times/ day, PO, for 7-10 days; or 24 hours prior to the procedure, give phenoxybenzamine $0.5 \mathrm{mg} / \mathrm{kg}$ in $250 c \mathrm{c}$ of D5W, slowly IV, over 2 hours

* Always administer supplemental oxygen with caution in a patient with chronic pulmonary disease. 
herniation, or congenital shortening of the pedicles represent an absolute contraindication to performing a interlaminar epidural injection at that level (6770). Subdural hematoma has also been reported following cervical epidural cortisone injection (71).

\section{Thoracic Interlaminar Epidural Injections}

Thoracic epidural techniques are similar to lumbar but the presence of the narrow epidural space and close proximity to the spinal cord in the thoracic vertebral canal makes spinal cord trauma more likely. The incidence of spinal cord damage is unknown, although the incidence of infection is increased in the thoracic spine when compared to the lumbar spine (72). The presence of the lungs on either side of the spine makes a pneumothorax a potential complication that is not usually a consideration with either a cervical or lumbar injection. The injection of local anesthetic in the mid thoracic epidural space may cause inhibition of the cardiac accelerator zone causing hypotension and bradycardia and its potential sequelae. In addition, a thoracic motor block from either the epidural or intrathecal injection of local anesthetic can cause up to $50 \%$ reduction in tidal volume making adequate ventilation of a patient with pulmonary disease difficult.

\section{Transforaminal Epidural Injection}

A transforaminal epidural injection is an epidural injection via the neural foramen. A spinal needle is typically directed into the foramen under fluoroscopic guidance. Complications may occur due to vascular injury, neurologic injury, or local anesthetic toxicity. Prior to entering the foramen, the needle may penetrate a variety of blood vessels, however the vertebral artery in the neck and radicular artery at any level are of particular interest $(33,73-81)$. The artery of Adamkiewicz is particularly vulnerable in the left upper lumbar spine or lower thoracic spine (73). It is important to know that this artery is variable in its position. Eighty percent of the time it enters the spine on the left from T8 to L1 however $20 \%$ of the time it is not in this position and may enter as low as L4 (82). If the needle penetrates the vertebral artery then there is risk of posterior circulation stroke from an embolic phenomenon or from clotting or occlusion of the artery. If local anesthetic is injected into the artery then the patient may have a seizure. Needle trauma with or without injection of an anterior or posterior radicular artery may cause an anterior or posterior spinal cord infarction. The needle may directly traumatize the spinal nerve, dorsal root ganglion, or spinal cord. And finally, if the needle penetrates the dural cuff and local anesthetic is injected spinal anesthesia may occur.

The authors $(83,84)$ are aware of multiple cases of paraplegia from apparent trauma to this artery during transforaminal injection. Some explanations include trauma to the spinal cord adjacent to the foramen being injected, the dural cuff injection causing spinal anesthesia, trauma of a posterior radicular artery causing a posterior cord stroke, trauma to the vertebral artery causing a cerebellar stroke, the vertebral artery injection causing seizure activity, and trauma to the spinal nerve causing a chronic cervical radiculopathy. Extreme caution must be exercised when performing this injection.

\section{Discography}

The most common severe complication after discography is infection of the disc, which is commonly referred to as discitis. Discitis following lumbar discography should occur no more frequently than 1 in 500 to 1 in 750 discs injected (85-89). The most common organisms infecting the lumbar disc are Staphylococcus aureus and Staphylococcus epidermidis (85-89). Occasionally a colonic organism involves the lumbar discs as a result of penetrating the colon with the discography needle. Because of the limited blood supply of the disc, such infections may prove difficult to eradicate. Discitis usually presents as an increase in spine pain 5 to 14 days following discography. Acutely, there should be no change in the patient's neurologic status. An elevated sedimentation rate will be seen within the first week to 10 days and bone scan turns positive at 14 - 33 days. Magnetic resonance imaging is now considered the gold standard in the detection of discitis, which was found to be superior to bone scanning with a $92 \%$ sensitivity, $97 \%$ specificity, and a $95 \%$ overall accuracy (90-92).

The incidence of thoracic discitis following a thoracic discogram is unknown but the organisms infecting those discs following discography should be similar to those involving the lumbar discs. Simi- larly, the incidence of pneumothorax and large vessel damage following thoracic discography is also unknown. In one series of 230 outpatient thoracic discograms, Schellhas (93) reported a zero incidence of pneumothorax. While this is encouraging, the complication does still occur and thus the procedure should not be attempted without substantial experience and training.

Cervical discitis is generally profound and life threatening $(94,95)$. The esophagus has gram-negative and anaerobic bacteria as components of its normal flora. Thus, placing the discography needle through it and into the disc may seed the disc with bacteria that may initiate a profound infection. In the mid and lower cervical spine, the esophagus lies on the left side of the larynx. The carotid sheath lies on the anterolateral surface of the cervical spinal column. As a result, a cervical discogram should be performed using a right paralaryngeal approach or an anterolateral approach (96). In performing the right paratracheal technique, the patient should be supine with the neck slightly extended. The esophagus should be pushed to the left and the carotid sheath to the right, thus minimizing the risk of trauma to these structures. The needle should be placed into the disc over the injectionist's superior finger maintaining the position of the esophagus and carotid sheath. In performing the anterolateral technique, the patient is positioned as described above, and the fluoroscopy unit is rotated to superimpose the uncus on the anterolateral superior body. The needle is advanced down to the uncus and then over the uncus and into the disc. The trajectory of the needle using this technique lies lateral to the carotid sheath and esophagus and anterior to the anterior rami and brachial plexus. If the needle penetrates the carotid sheath then direct injury to the vagus nerve or carotid artery could occur with its associated risks and complications.

In addition to infectious complications, pneumothorax may occur after cervical and thoracic discography. This complication should rarely occur if appropriate technique is used. Most pneumothoraces following cervical or thoracic discography are small (10 - 15\% of lung volume) and can often be treated conservatively, however, all pneumothoraces must be taken seriously and watched overnight with serial chest X-rays and close moni- 
toring of vital signs and blood gases. If the pneumothorax advances beyond 25\% of lung volume or if the patient's ability to oxygenate their blood is compromised then a chest tube must be placed (41).

Direct trauma to the anterior rootlets and spinal cord can occur if the needle is allowed to traverse the entire disc or is placed too laterally during cervical discography. Injury to the vertebral artery may occur during the anterolateral cervical discographic approach. Injury to the anterior ramus, spinal nerve, or dorsal root ganglion may occur during thoracic or lumbar discography if the needle is advanced towards the disc too superior or too lateral in the "safe triangle" within the foramen $(97,98)$. These complications should rarely occur if appropriate technique and precautions are used. Such needle-induced trauma to the cervical spinal cord can result in syrinx formation with attendant progressive neurologic deficit, including quadriplegia.

\section{Intercostal Nerve Blocks}

Given the proximity of the pleural space, pneumothorax after intercostal nerve blocks is a distinct possibility. The incidence of the complication is between $0.08 \%$ and $1.4 \%$ per nerve blocked (99), but occurs with greater frequency in patients with chronic obstructive pulmonary disease. Because of the proximity to the intercostal nerve and artery, when analgesia is produced from the intercostal block, the compensatory vasoconstriction eases and the patient may become hypotensive. In a similar manner, intercostal blocks can lead to respiratory failure, when pain relief from the block unmasks the ventilatory depression of previously administered but ineffective parenteral narcotics (100).

\section{Facet Joint Block}

The most often cited problem is a transient exacerbation in pain (about $2 \%$ incidence) lasting as long as 6 weeks to 8 months in some cases (101). Spinal anesthesia may occur after facet joint injection if the needle is positioned within the thecal sac or if there is an abnormal communication between the facet joint capsule and the thecal sac. Chemical meningitis after lumbar facet block has been reported (101-103). Both of these complications are thought to have occurred after inadvertent dural puncture. Facet capsule rupture also occurs, especially if more than $2.0 \mathrm{~mL}$ of injectate is used for intraarticular injections.

During performance of cervical facet blockade, there is the potential risk of entry into the intervertebral foramen, spinal canal, and vertebral artery. These complications occur more frequently using a lateral intra-articular technique than with blockade of the medial branches innervating the cervical facet joints because the former technique requires deeper penetration of the needle into the joint and toward the spinal structures. Local anesthetic may leak out of the joint into these areas, causing motor and sensory blockade with its attendant risks and complications.

Third occipital nerve blocks can cause transient ataxia and unsteadiness due to partial blockade of the upper cervical proprioceptive afferents and the righting response $(104,105)$. In one study of cervical facet joint radiofrequency denervation, $13 \%$ of the patients complained of post-procedure pain that resolved in 2 to 6 weeks and $4 \%$ complained of occipital hypesthesia probably due to a lesion of the third occipital nerve, which resolved in 3 months (105). No persistent motor or sensory deficits occurred.

\section{Sympathetic Nerve Blocks}

In the cervicothoracic (stellate ganglion) block, acute potentially life-threatening complications may occur including seizures, spinal block, hypotension, or pneumothorax (106-113). Additional complications include block or injury to the recurrent laryngeal nerve, phrenic nerve, sympathetic trunk, apex of the lung, or brachial plexus.

In the lumbar sympathetic block, 6. potential complications include intravascular injections, intradural injections with spinal anesthesia or postural headaches, hypotension, lumbar plexus block, renal puncture, or genitofemoral neuralgia $(107,112,113)$. Other risks include injury to the spleen, gut, liver, and injection of large volumes of local anesthetic into the aorta or inferior vena cava.

\section{Author Affiliation:}

Robert E. Windsor, MD

Program Director \& President Georgia Pain Physicians, PC

Emory University

2550 Windy Hill Road, Suite 215

Atlanta, GA 30067

E-mail: rwindsor@aol.com

\section{Seneca Storm, MD}

Fellow

Georgia Pain Physicians, PC

Emory University

2550 Windy Hill Road, Suite 215

Atlanta, GA 30067

E-mail: senecastorm@hotmail.com

\section{Ross Sugar, MD}

Faculty

Georgia Pain Physicians, PC

Emory University

2550 Windy Hill Road, Suite 215

Atlanta, GA 30067

\section{RefERENCES}

1. Falco F. Lumbar spine injection procedures in the management of low back pain. Occup Med 1998; 13:121-149.

2. Kinard R: Diagnostic spinal injection procedures. Neurosurg Clin N Am 1996; 7: 151-165.

3. Manchikanti L, Singh V. Interventional pain management: Evolving issues for 2003. Pain Physician 2003; 6:125-137.

4. Moskovich R. Epidural injections for the treatment of low back pain. Bull Hosp Jt Dis 1996; 55:178-184.

5. Sunderland S. Nerves and Nerve Injuries. Ed 2. Edinburgh, Churchill Livingston, 1978.

Messerli F, Grossman E. The use of sublingual nifedipine: a continuing concern. Arch Intern Med 1999; 19:2259-2260.

7. Dougherty J, Fraser R. Complications following intraspinal injections of steroids: Reports of two cases. J Neurosurg 1978; 48:1023-1025.

8. Gutknecht D. Chemical meningitis following epidural injection of corticosteriods. Am J Med 1987; 82:570.

9. Shealy C. Dangers of spinal injections without proper diagnosis. JAMA 1966; 197:1104-1106.

10. Goucke C, Graziotti P. Extradural abscess following local anesthetic and steroid injection for chronic low back pain. $\mathrm{Br} / \mathrm{An}$ aesth 1990; 65:427-429.

11. Chan S, Leung S. Spinal epidural abscess following steroid injection for sciatica. 
Case report. Spine 1984; 14:106-108.

12. Tham E, Stoodley M, Macintyre P, et al. Back pain following postoperative epidural analgesia: An indicator of possible infection. Anesth Intensive Care 1997; 25: 297-301.

13. Cooper A, Sharpe M et al. Bacterial meningitis and cauda equina syndrome after epidural steroid injections. Can J Anaesth. 1996; 43:471-474.

14. Baker A, Ojemann R, Swartz M et al. Spinal epidural abscess. NEJM 1975; 293: 463-468.

15. Bonica J. Diagnostic and therapeutic blocks. A reappraisal based on 15 years experience. Anesth Analg Curr Res 1958; 37:58.

16. Furman M, Giovanniello M, O’Brien E. Incidence of intravascular penetration in transforaminal cervical epidural steroid injections. Spine 2003; 28:21-25.

17. Furman MB, O’Brien EM, Zgleszewski TM. Incidence of intravascular penetration in transforaminal lumbosacral epidural steroid injections. Spine 2000; 25: 26282632.

18. Odom J, Sih I. Epidural analgesia and anticoagulant therapy: Experience with one thousand cases of continuous epidurals. Anesthesia 1983; 38:550-551.

19. Rao T, El-Etr A. Anticoagulation following placement of epidural and subarachnoid catheters. Anesthesiology 1981; 55:618620.

20. Delaney T, Rowlingson J, Carron $\mathrm{H}$ et al. Epidural steroid effects on nerves and meninges. Anaesth Analg Curr Res 1980; 59: 610-614.

21. Knight C, Burnell J. Systemic side effects of extradural steroids. Anaesthesia 1980; 35:593-594.

22. Goebert H. Sciatica: Treatment with injections of procaine and hydrocortisone acetate. Results in 113 patients. Anesth Analg 1960; 130-134.

23. Knutsen O, Ygge H. Prolonged extradural anesthesia with bupivacaine at lumbago and sciatica. Acta Orthop Scand 1971; 42: 338-352.

24. Stoll A, Sanchez M. Epidural Hematoma after epidural block: Implications for its use in pain management. Surg Neurol 2002; 57:235-40.

25. American Heart Association. Textbook of advanced cardiac life support. 3rd Ed. 1993.

26. American Heart Association. Guidelines for Cardiopulmonary Resuscitation and Emergency Cardiac Care. JAMA 1992; 268: 2171-2302.

27. Housholder-Hughes S. Advanced cardiac life support for the new millennium. J Cardiovasc Nurs 2002; 16:9-23.

28. Holtkamp M, Masuhr F, Harms L. The management of refractory generalized convulsive and complex partial status epilepticus in three European countries: A survey among epileptologists and critical care neurologists. J Neurol Neurosurg Psychiatry $2003 ;$ 74:1095-1099.

29. Outin H, Liot P, De Jonghe B et al. Management of adult refractory convulsive status epilepticus in the intensive care unit. Rev Neurol 2002; 158:1059-1068.

30. Lacey D. Status epilepticus in children and adults. J Clin Psychiatry 1988; 49:33-36.

31. White A, Derby R, Wynne G. Epidural injections for the diagnosis and treatment of low-back pain. Spine 1980; 5:78-86.

32. El-Khoury G, Ehara S, Weinstein J et al. Epidural steroid injection: a procedure ideally performed with fluoroscopic control. Radiology 1988; 168:554-557.

33. Baker R, Dreyfuss P, Mercer $S$ et al. Cervical transforaminal injection of corticosteroids into a radicular artery: A possible mechanism for spinal cord injury. Pain 2003; 103:211-215.

34. Renfrew D, Moore T, Kathol M et al. Correct placement of epidural steroid injections: Fluoroscopic guidance and contrast administration. A/NR 1991; 12:1003-1007.

35. Brouwers $P$, Kottink E, Simon $M$ et al. A cervical anterior spinal artery syndrome after diagnostic blockade of the right C6nerve root. Pain 2001; 91:397-399.

36. Rawal N, Wattwil M. Respiratory depression following epidural morphine: An experimental and clinical study. Anesth Analg 1984; 63:8-14.

37. Rawal N, Arner S, Gustafsson L et al. Present state of epidural and intrathecal opiate analgesia: A nationwide follow-up survey in Sweden. Br J Anaesth 1987; 59:791799.

38. Arner S, Rawal N, Gustafsson LL. Clinical experience of long-term treatment with epidural and intrathecal opioids--a nationwide survey. Acta Anaesthesiol Scand 1988; 32:253-259.

39. Rawal N, Sjostrand U. Clinical application of epidural and intrathecal opioids for pain management. Int Anesthesiol Clin 1986; 24:43-57.

40. Brindenbaugh PO. Post-operative intercostal nerve block analgesia versus narcotic analgesia. Anesth Analg 1973; 52:

41. Idrees $M$, Ingleby A, Wali S. Evaluation and management of pneumothorax. Saudi Med / 2003; 24:447-452.

42. Armitage EN. Lumbar and thoracic epidural. In Wildsmith JAW, Armitage EN (eds). Principles and Practice of Regional Anesthesia. New York, Churchill Livingstone, 1987, p 109.

43. Bromage PR. Complications and contraindications. In Bromage PR (ed). Epidural Analgesia. Philadelphia, WB Saunders, 1978, pp 654-711.

44. Benzon $H$. Epidural steroid injections for low back pain and lumbosacral radiculopathy. Pain 1986; 24:277-295.

45. Swerdlow M, Sayle-Creer W. A study of extradural medication in the relief of the lumbosciatic syndrome. Anaesthesia
1970; 25:341-345.

46. Warr A, Wilkinson J, Burn J et al. Chronic lumbosciatic syndrome treated by epidural injection and manipulation. Practitioner 1972; 299:53-59.

47. Kepes E, Duncalf D. Treatment of low backache with spinal injections of local anesthetics, spinal and systemic steroids: A review. Pain 1985; 22:33-47.

48. Deisenhammer E. Clinical and experimental studies on headaches after myelography. Neuroradiology 1985; 9:99-102.

49. Waldman S. Subdural injection as a cause of unexplained neurological symptoms. Reg Anesth 1992; 17:55.

50. Mehta M, Salmon N. Extradural block. Confirmation of the injection site by $x$-ray monitoring. Anesth 1985; 40:1009-1012.

51. Singh V, Manchikanti L. Role of caudal epidural injections in the management of chronic low back pain. Pain Physician 2002; 5:133-148.

52. Manchikanti L, Staats P, Singh V et al. Evidence-based practice guidelines for interventional techniques in the management of chronic spinal pain. Pain Physician 2003; 6:3-80.

53. Bogduk N. Epidural steroids for low back pain and sciatica. Pain Digest 1999; 9: 226-227.

54. White A: Injection techniques for the diagnosis and treatment of low back pain. Orthop Clin North Am 1983;14:553-567.

55. Botwin KP, Thomas S, Gruber RD et al. Radiation exposure of the spinal interventionalist performing fluoroscopically guided lumbar transforaminal epidural steroid injections. Arch Phys Med Rehabil 2002; 83:697-701.

56. Manchikanti L. Transforaminal lumbar epidural steroid injections. Pain Physician 2000; 3:374-398.

57. Botwin KP, Freeman ED, Gruber RD et al. Radiation exposure to the physician performing fluoroscopically guided caudal epidural steroid injections. Pain Physician 2001; 4:343-348.

58. Manchikanti L, Pampati V. Research designs in interventional pain management Is randomization superior, desirable or essential? Pain Physician 2002; 5:275-284.

59. Manchikanti LM, Cash KA, Moss TL et al Radiation exposure to the physician in interventional pain management. Pain Physician 2002; 5:385-393.

6o. Manchikanti L, Cash K, Moss T, Pampati V. Effectiveness of protective measures in reducing risk of radiation exposure in interventional pain management: A prospective evaluation. Pain Physician 2003; 6: 301-306.

61. Simon D, Borelli S, Braathen L et al. Allergic or pseudoallergic reaction following epidural steroid deposition and skin testing. Reg Anesth 1989; 14:253-255.

62. Covino BG. Clinical pharmacology of local anesthetic agents. In Cousins MJ, Bridenbaugh MJ, Phillip O (eds): Neural Block- 
ade in Clinical Anesthesia and Management of Pain. Philadelphia, Lippincott, 1988, pp 111-144.

64. Braid D, Scott D. Dosage of lignocaine in epidural block in relation to toxicity. $\mathrm{Br} J$ Anaesth 1966; 38:596-602.

65. Cousins M. Epidural neural blockade. In Cousins MJ, Bridenbaugh MJ, Phillip O (eds): Neural Blockade in Clinical Anesthesia and Management of Pain. Philadelphia, Lippincott, 1988, pp 340-341.

66. Cousins $M$. Hematoma following epidural block. Anesthesiology 1972; 37:263.

67. Lerner S, Gutterman P, Jenkins F. Epidural hematoma and paraplegia after numerous lumbar punctures. Anesthesia 1973; 39: 550-553.

68. Waldman S. Cervical epidural steroid nerve blocks: A prospective study of complications occurring during 790 consecutive blocks. Reg Anesth 1989; 11:149-152.

69. Botwin K, Castellanos R, Rao S et al. Com plications of fluoroscopically guided interlaminar cervical epidural injections. Arch Phys Med Rehabil 2003; 84:627-633.

70. Hodges S, Castleberg R, Miller T et al. Cervical epidural steroid injection with intrinsic spinal cord damage: Two case reports. Spine 1998; 23:2137-2140.

71. Reitman C, Watters W. Subdural hematoma after cervical epidural steroid injection. Spine 2002; 27:E174-E176.

72. Redekop G, Del Maestro R. Diagnosis and management of spinal epidural abscess. Can J Neurol Sci 1992; 19:180-187.

73. Alleyne C, Cawley C, Shengelaia G et. al. Microsurgical anatomy of the artery of Adamkiewicz and its segmental artery. J Neu rosurg 1998; 89:791-795.

74. Manchikanti L. Cervical epidural steroid injection with intrinsic spinal cord damage. Spine 1999; 24:1170-1172.

75. Nelson JW. Letter to the Editor. In response to Houten JK, Errico TJ. Paraplegia after lumbosacral nerve block. Spine J 2003; 2 : 88-89.

76. Houten JK, Errico TJ. Paraplegia after lumbosacral nerve root block: Report of three cases. Spine / 2002; 2:70-75

77. Helm II S, Jasper JF, Racz GB. Complications of transforaminal epidural injections. Pain Physician 2003; 6:389-390.

78. Schultz D. Risk of transforaminal epidural injections. Pain Physician 2003; 6:390.

79. Kloth D. Risk of cervical transforaminal epidural injections by anterior approach. Pain Physician 2003; 6:392-393.

80. Jasper J. Role of digital subtraction fluoroscopic imaging in detecting intravascular injections. Pain Physician 2003; 6:369372.
81. Botwin KP, Gruber RD, Bouchlas CG et al. Complications of fluoroscopically guided transforaminal lumbar epidural injections. Arch Phys Med Rehabil 2003; 81:10451050.

82. Lo D, Vallee J, Spelle L et al. Unusual origin of the artery of Adamkiewicz from the fourth lumbar artery. Neuroradiology 2002; 44:153-157.

83. Windsor R, Falco, F, Dalton L. A suggested modification to the transforaminal injection procedure in the upper lumbar and lower thoracic spine. ISIS Newsletter, 2000.

84. Windsor R, Overton A, Sugar R. Cervical transforaminal injection. Three case reports detailing complications, a review of the literature, and a suggested technique. Pain Physician 2003; 6:457-465.

85. Guyer R, Collier R, Stith W et al. Discitis after discography. Spine 1988; 13:13521354 .

86. Fernand R, Lee C. Postlaminectomy disc space infection. A review of the literature and a report of three cases. Clin Orthop 1986; 209:215-218.

87. Schulitz K, Assheuer J. Discitis after procedures on the intervertebral disc. Spine 1994; 19:1172-1177.

88. Thibodeau A. Closed space infection following the removal of lumbar intervertebral discs. J Bone Joint Surg 1968; 50A: 400-410.

89. Crock H. Practice of spinal surgery. New York, Springer-Verlag, 1983.

90. Arrington J, Murtagh F, Silbiger $\mathrm{M}$ et al. MRI of post-discogram discitis and osteomyelitis in the lumbar spine: Case report. J Fla Med Assoc 1986; 73:192-194.

91. Modic M, Feiglin D, Piraino D et al. Vertebral osteomyelitis: Assessment using MR. Radiology 1985;157:157-166.

92. Szypryt E, Hardy J, Hinton C et al. A comparison between $\mathrm{MRI}$ and scintigraphic bone imaging in the diagnosis of disc space infection in an animal model. Spine 1988; 13:1042-1048.

93. Schellhas K, Pollei K. Thoracic discography. A safe and reliable technique. Spine 1994; 19:2103-2109.

94. Zeidman S, Thompson K, Ducker T. Complications of cervical discography: Analysis of 4400 diagnostic disc injections. Neurosurgery 1995; 37:414-417.

95. Guyer R, Ohnmeiss D, Mason S. Complications of cervical discography: Findings in a large series. J Spinal Disord 1997; 10:95101

96. Distelmaier P, Lins E. Technique and value of discography for the cervical syndrome.
Rontgenblatter 1976; 29:178-183

98. Saal J. General principles of diagnostic testing as related to painful lumbar spine disorders: A critical appraisal of current diagnostic techniques. Spine 2002; 27: 2538-2545.

98. Kumar N, Agorastides I. The curved needle technique for accessing the $\mathrm{L}_{5} / \mathrm{S}_{1}$ disc space. Br J Radiol 2000; 73:655-657.

99. Shanti C, Carlin A, Tyburski J. Incidence of pneumothorax from intercostal nerve block for analgesia in rib fractures. J Trauma 2001; 51:536-539.

100. Cory P, Mulroy M. Post-operative respiratory failure following intercostal block. Anesthesiology 1981; 54:418-419.

101. Bous R. Facet joint injections. In Stanton-Hicks M, Bous R (eds): In Chronic Low Back Pain. New York, Raven Press, 1982, pp 199-211.

102. Thomson SJ, Lomax DM, Collett BJ. Chemical meningism after lumbar facet joint nerve block with local anesthetic and steroids. Anesthesia 1991; 46:563-564.

103. Berrigan T: Chemical meningism after lumbar facet joint block. Anesthesia 1992; 47:905-906.

104. Pawl RP. Headache, cervical spondylosis, and anterior cervical fusion. Surg Ann 1971; 9:391-408.

105. Bogduk N, Marsland A. The cervical zygapophysial joints as a source of neck pain. Spine 1988; 13:610-617.

106. Carron H, Litwiller R. Stellate ganglion block. Anesth Analg 1975; 54:567.

107. Lofstrom J. Sympathetic neural blockade of upper and lower extremity. In Cousins M (ed). Neural Blockade in Clinical Management of Pain, $2^{\text {nd }} \mathrm{Ed}$. Philadelphia, Lippincott, 1988, p 461.

108. Melis M, Zawawi K, al-Badawi $\mathrm{E}$ et al. Complex regional pain syndrome in the head and neck: a review of the literature. I Orofac Pain 2002; 16:93-104.

109. Saches B, Zindrick M, Beasley R. Reflex sympathetic dystrophy after operative procedures on the lumbar spine. J Bone Surg 1993; 75A:721-724.

110. Horton W. Reflex sympathetic dystrophy after operative procedures on the lumbar spine. J Bone Joint Surg Am 1994; 76:1113.

111. Wallace M, Milholland A. Contralateral spread of local anesthetic with stellate ganglion block. Reg Anesth 1993; 18:5559.

112. Schmidt S, Gibbons J. Postdural puncture headache after fluoroscopically-guided lumbar paravertebral sympathetic block. Anesthesiology 1993; 78:198.

113. Sprague R, Ramamurthy S. Identification of the anterior psoas sheath as a landmark for lumbar sympathetic block. Reg Anesth 1990; 15:253. 
